Research Paper

\title{
Comprehensive investigation of p53, p21, nm23, and VEGF expression in hepatitis $B$ virus-related hepatocellular carcinoma overall survival after hepatectomy
}

\author{
Guang-Zhi Zhu1 ${ }^{*}$, Xi-Wen Liao ${ }^{1 *}$, Xiang-Kun Wang1, Yi-Zhen Gong², Xiao-Guang Liu' ${ }^{1}$, Long Yu1, \\ Chuang-Ye Han ${ }^{1}$, Cheng-Kun Yang1, Hao Su${ }^{1}$, Ke-Tuan Huang1, Ting-Dong Yu1, Jian-Lu Huang 1,3, Jia Li, \\ Zhi-Ming Zeng5, Wei Qin¹, Zheng-Qian Liu1, Xin Zhou¹, Jun-Qi Liu1, Lei Lu1, Quan-Fa Han¹, Li-Ming \\ Shang ${ }^{1}$, Xin-Ping Ye ${ }^{1}$, Tao Peng ${ }^{1 凶}$ \\ 1. Department of Hepatobiliary Surgery, The First Affiliated Hospital of Guangxi Medical University, Nanning, 530021, Guangxi Zhuang Autonomous \\ Region, People's Republic of China. \\ 2. Department of Colorectal and Anal Surgery, The First Affiliated Hospital of Guangxi Medical University, Nanning, 530021, Guangxi Zhuang Autonomous \\ Region, People's Republic of China. \\ 3. Department of Hepatobiliary Surgery, The Third Affiliated Hospital of Guangxi Medical University, Nanning, 530031, Guangxi Zhuang Autonomous \\ Region, People's Republic of China. \\ 4. Department of Pathology, First Affiliated Hospital of Guangxi Medical University, Nanning, Guangxi Zhuang Autonomous Region 530021, People's \\ Republic of China. \\ 5. Department of Medical Oncology, First Affiliated Hospital of Guangxi Medical University, Nanning, Guangxi Zhuang Autonomous Region 530021, \\ People's Republic of China. \\ ${ }^{*}$ Contributed equally
}

$\square$ Corresponding author: Prof. Tao Peng, Department of Hepatobiliary Surgery, The First Affiliated Hospital of Guangxi Medical University, Nanning, 530021, Guangxi Zhuang Autonomous Region, People's Republic of China. Tel: (+86)-771-5356528. Fax: (+86)-771-5350031. E-mail: pengtaogmu@163.com; pengtao@gxmu.edu.cn. ORCID ID: https://orcid.org/0000-0001-6133-7078.

(c) The author(s). This is an open access article distributed under the terms of the Creative Commons Attribution License (https://creativecommons.org/licenses/by/4.0/). See http://ivyspring.com/terms for full terms and conditions.

Received: 2019.02.01; Accepted: 2019.09.18; Published: 2020.01.01

\begin{abstract}
Objective: The goal of our current study is to assess the immunohistochemical of p53, p21, nm23, and VEGF expression in hepatitis B virus (HBV)-related hepatocellular carcinoma $(\mathrm{HCC})$ prognosis after hepatectomy, as well as the prospective molecular mechanisms of prognostic indicator.

Methods: There were 419 HBV-related HCC patients who were from southern China of Guangxi province and were used to evaluate the immunohistochemical expression for these biomarkers in prognosis. A genome-wide expression microarray dataset of HBV-related HCC were obtained from GSE14520.

Results: In our study, the expression of $\mathrm{p} 53, \mathrm{p} 21$, and nm23 in cancer tissues of patients with hepatitis B-related hepatocellular carcinoma did not affected the clinical outcome of 2 years, 5 years or overall. Patients with high expression of VEGF had a worse overall survival after 2 years of surgery than patients with low expression (adjusted $P=0.040$, adjusted $H R=1.652,95 \% \mathrm{Cl}=1.024-2.665$ ). Survival analysis of VEGF in GSE14520 cohort also demonstrated that VEGF mRNA expression also significantly associated with $\mathrm{HBV}$-related HCC OS (adjusted $P=0.035$, adjusted $H R=1.651,95 \% \mathrm{Cl}=1.035-2.634$ ). The prospective molecular mechanisms by co-expression analysis suggested that VEGF might be correlated to regulation of cell proliferation, cell growth and apoptotic process, Rapl signaling pathway, HIF-1 signaling pathway, PPAR signaling pathway, cell cycle. Whereas the GSEA suggested that VEGF might involve in the regulation of HIF and HIFIA pathway, and TP53 regulation pathway.

Conclusion: Our findings suggested that VEGF might be a prognostic indicator of HBV-related HCC, and we also identified the VEGF prospective molecular mechanisms through the whole genome co-expression and GSEA approaches.
\end{abstract}

Key words: hepatitis B virus, hepatocellular carcinoma, overall survival, immunohistochemical, hepatectomy 


\section{Introduction}

Liver cancer is ranked as one of the top common malignant tumors around the globe. Merely in the year of 2012, over 780 thousand new cases of liver cancer were diagnosed each year in the world, with China $50 \%$ of the total number of cases [1]. The annual incidence of liver cancer in China was 370,000 $(27.29 / 100,000)$ with the death rate 310,000 $(23.76 / 100,000)$, and ranked fourth in the third malignant tumor spectrum and the death spectrum respectively [2]. Most (70\% to 90\%) liver cancers occurring worldwide are hepatocellular carcinoma (HCC) [3]. There are many factors contributing to the development of HCC, including alcohol abuse, Aflatoxin-1 and hepatitis B virus (HBV), nonalcoholic fatty liver disease (NAFLD) and hepatitis $C$ virus (HCV) infection [4, 5]. In Guangxi, the male and female liver cancer mortality was $69.0 / 100,000$ and $17.9 / 100,000$ respectively, which was the highest fatality rate for male and female patients in China [6]. Epidemiological studies showed that the major risk factors for liver cancer in Guangxi included three major risk factors: hepatitis viruses (especially HBV), aflatoxin (AFB) intake, and drinking water source pollution [7-10].

Even if the HCC patients after surgical resection or liver transplantation, the prognosis of HCC was still not satisfactory. The prognosis of liver cancer is affected by many clinical characteristics. Clinical characteristics such as vascular invasion, Barcelona Clinic Liver Cancer (BCLC) staging, tumor size, alpha-fetal protein (AFP), morphological and pathological features are traditionally the most important prognostic factors. Those related studies that had be conducted before had shown that the expression of p53 [also known as tumor protein p53 (TP53)], p21 [cyclin dependent kinase inhibitor 1A (CDKN1A)], nm23 [also known as nucleoside diphosphate kinase 1 (NME1)] and VEGF [also known as vascular endothelial growth factor A (VEGFA)] could reflect the prognosis of liver cancer by immunohistochemical techniques [11-14]. p53 protein, a protein suppressing tumor, has response to diverse cellular stresses for regulating the expression of target genes, and thus induces senescence, apoptosis, cell cycle arrest, DNA repair, or changes in metabolism. p21, being a potent cyclin-dependent kinase inhibitor, performs as a regulator of cell cycle progression at G1 for it not only binding to but also inhibiting the activity of cyclin-cyclin-dependent kinase2 or cyclin-dependent kinase 4 complexes. VEGF, a heparin-binding protein, induces proliferation and migration of vascular endothelial cells and is vital to both physiological and pathological angiogenesis. This gene, whose expression is related to tumor stage and progression, is unregulated in many identified tumors. nm23, a suppressor for tumor metastasis, has regulation function towards a variety of cellular activities, which includes proliferation, differentiation, migration and apoptosis. Recent studies had shown the common understanding that the cell-cycle proteins could have interaction with $\mathrm{nm} 23$ and might function as modulators of the metastasis suppressor activity [15]. Previous studies had shown different views about these immunohistochemical markers on prognosis, which might be the result of a different background in the research population of these studies. Guangxi is a highly exposed area of HBV. In this study, the expression of p53, p21, nm23, VEGF protein in tumor tissue of HBV-related HCC patients within Guangxi combined with other markers (such as AFP, BCLC stage, tumor size) were analyzed for estimating the prognostic value of patients after HCC resection.

\section{Methods}

\section{Study population}

The Ethics Committee of the First Affiliated Hospital of Guangxi Medical University had grant the approval for this study. We examined a total of 419 cases from the patients with HCC whose clinical characteristics from 2003 to 2013 were collected from the First Affiliated Hospital of Guangxi Medical University, Guangxi, China. All sample serological tests were positive for hepatitis B surface antigen (HBsAg) and histopathology were confirmed to be hepatocellular carcinoma. The tumor status was categorized by the BCLC staging system, and the liver function was identified according to the Child-Pugh classification. Portal vein tumor thrombus (PVTT) was identified in accordance with the previous study [16]. The follow-up time of the patients was after surgery until death or the final follow-up which was conducted in September 2014.

\section{Immunohistochemical and scoring}

All HCC samples were obtained during operation and stored right away at $-80 \mathrm{C}$ for further application. Tissue blocks prepared from HCC tissues were used to perform p53, p21, VEGF and nm23 immunohistochemistry (IHC). To be brief, all the specimens were cut off by formalin fixation and paraffin embedding, and triethylene-propyl triethoxysilane was processed into slices. The slices were routinely dewaxed and hydrated and washed in ethanol. Tissue immunohistochemical staining was conducted by the manufacturer's instructions. The sections were incubated by primary antibody (anti-p53, anti-p21, anti-nm23, anti-VEGF, at Vitrogen, Camarillo, CA) for 1 hour $37^{\circ} \mathrm{C}$. The working dilution 
of the primary antibody was 1:50. The slice was firstly PBS washed $\mathrm{g}$ for 15 minutes, then incubated with ENVISION+ rabbit/horseradish peroxidase for 45 minutes, and finally 15 minutes after Peroxidase. The positive and negative controls were performed on each section. Instead of the primary antibody, normal rabbit serum IgG was used as negative control. We conducted all experiments in duplicate.

p21 and p53 immunostaining was estimated quantitatively by counting the total number of positively stained nuclei per 10 high-power fields ( $\times 400$ magnification) microscopically from the slides. We found that only nuclear staining was positive for p21 and p53. Established on the previously published criteria, positive staining of p21 and p53 was identified when $>5 \%$ of tumor cells were stained [17, 18]. The cases were considered positive to $\mathrm{nm} 23$ and VEGF protein expression if more than $10 \%$ of the tumors cells showed cytoplasm of tumor cells staining, as performed in previous studies $[19,20]$. Our research observed the stained sections under a light microscope (400×) (Olympus, Japan). Two independent pathologists confirmed the clinicopathological features of these patients. The mean percentage value of two cores was taken as the representative of one tumor, and discrepancies were resolved by consensus.

\section{Validation cohort at mRNA level and bioinformatics analysis}

To verify the prognostic values of TP53, NME1, VEGFA and CDKN1A at mRNA level, GSE14520 (http:// www.ncbi.nlm.nih.gov/geo/query/acc.cgi?a $\mathrm{cc}=\mathrm{GSE} 14520$ ), a genome-wide expression microarray dataset with HBV-related HCC was serve as validation cohort. The detailed procedure of data processing could be found in our previous studies [21]. Then the prospective molecular mechanism of prognostic indicators of $\mathrm{HBV}$-related HCC were investigated by gene set enrichment analysis (GSEA, http://software.broadinstitute.org/gsea/index.jsp) with the reference gene set from Molecular Signatures Database (MSigDB) gene sets: c2 gene set (c2.all.v6.1. symbols.gmt) and c5 gene set (c5.all.v6.1.symbols. gmt) [22] [23]. In addition, genome-wide coexpression analysis to identified co-expression genes of the prognostic genes were used to investigated the potential biological processes and pathways that associated with prognostic genes in HBV-related HCC tumor tissues. The potential biological processes and pathways were identified by applying the Database for Annotation, Visualization and Integrated Discovery v6.8 (DAVID v6.8, https://david.ncifcrf. gov/home.jsp) [24] [25] and Biological Networks Gene Ontology tool (BiNGO) in Cytoscape version
3.6.1 [26].

\section{Statistical analysis}

Statistical analysis was to explore the relationship between the clinical parameters of gender, age, tumor size, number of tumors, pathologic of grade, serum level of AFP, and the 4 immunohistochemical markers by chi-square test. Survival analysis was assessed by the Kaplan-Meiercurve with the log-rank test. Overall survival (OS) was defined from the date of follow-up (September 1,2014). Univariate analysis, which was conducted to explore the relationship between clinical features and survival analysis, was applied to calculate the crude and those result with $P<0.1$ were fitted into the Cox proportional hazards regression model. Cox proportional hazards regression analysis was used to calculate adjusted hazard ratio (HR) and 95\% confidence interval (CI) SPSS version 18.0 (SPSS, Inc., Chicago, IL, US) for Windows was applied for the statistical analyses. A value of $\mathrm{P}<0.05$ was taken as statistically significant.

\section{Results}

\section{Correlation analysis of immunohistochemical expression of p53, p21, nm23 and VEGF with clinicopathological characteristics.}

The expression of p53, p21, nm23, and VEGF in the 419 HCC cases were analyzed by IHC. The immunostaining results showed that 255 cases were positive and 164 cases were negative for p53, 112 cases were positive and 307 cases were negative for p21, 376 cases were positive and 43 cases were negative for nm23, 320 cases were positive and 99 cases were negative for VEGF, respectively. Age, tumor size, cirrhosis, and antiviral therapy were significantly associated with p21 expression $\left(\chi^{2}=5.722, P=0.017\right.$; $\chi^{2}=4.358, \quad P=0.037 ; \quad \chi^{2}=9.576, \quad P=0.002 ; \quad \chi^{2}=12.564$, $P<0.001$; respectively, Table 1). Antiviral therapy was closely correlative with $\mathrm{nm} 23$ expression $\left(\chi^{2}=6.791\right.$, $P=0.009)$. Race was considerably connected with p53 expression $\left(\chi^{2}=5.014, P=0.025\right)$. Smoking status was significantly associated with VEGF expression $\left(\chi^{2}=3.886, P=0.049\right)$. As demonstrated in Figure 1, p53 and p21 were mainly located in the nuclei of the cancer cell. nm23 while VEGF mainly in the cytoplasm of the cancer cell.

The median follow-up duration was 36.7 months, and the median survival time (MST) was 51 months. The distribution of clinical features in 419 patients with hepatocellular carcinoma was shown in Table 2. The gender, age, race, smoking status, BMI, AFP level, Child-Pugh, cirrhosis, and pathological grade were not notably associated with OS. However, 
the overall survival time was associated with alcohol status, BCLC status, portal vein tumor thrombus, antiviral therapy, tumor size, and tumor number (Log-rank $P$ value for drinking status $=0.043$, for tumor size $<0.001$, for tumor number $=0.001$, for PVTT $<0.001$, for antivirus therapy $=0.020$ ). Drinking patients had a higher risk of death than those who do not drink ( $\mathrm{HR}=1.335,95 \% \mathrm{CI}=1.066-1.770)$; patients with BCLC B or C stage had a higher risk of death than BCLC A stage patients (HR=1.880, 95\% $\mathrm{CI}=1.281-2.758 ; \quad \mathrm{HR}=2.766, \quad 95 \% \quad \mathrm{CI}=2.021-3.786$; respectively); multiple tumor patients had a higher risk of death than single tumor patients $(\mathrm{HR}=1.642$, 95\% CI=1.219-2.212); patients with a tumor size greater than $5 \mathrm{~cm}$ had a higher risk of death than patients with tumor size $\leq 5 \mathrm{~cm} \quad(\mathrm{HR}=1.981$, 95\% CI=1.416-2.770); patients with portal thrombosis had a higher risk of death than those without portal thrombosis $(\mathrm{HR}=2.801,95 \% \mathrm{CI}=2.025-3.875)$, and anti-HBV virus was less death risk than antiviral death $(\mathrm{HR}=0.675,95 \% \mathrm{CI}=0.483-0.945)$.

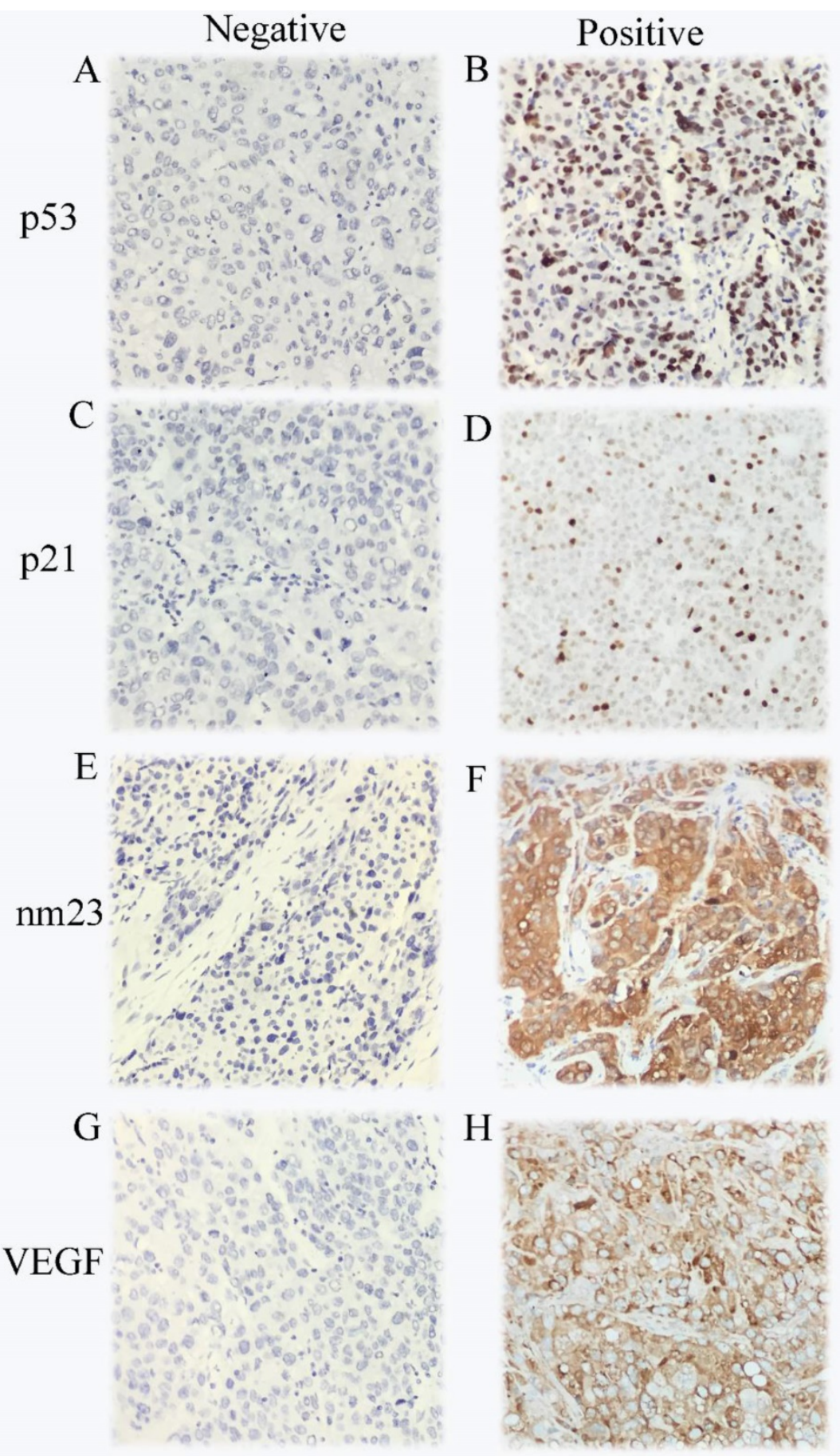

Figure 1. Immunohistochemical staining in HCC samples for p53 (A-B), p21 (C-D),nm23 (E-F) and VEGF (G-H). 
Table 1. Correlation analysis of immunohistochemical expression of p53, p21, nm23 and VEGF with clinicopathological data.

\begin{tabular}{|c|c|c|c|c|c|c|c|c|c|c|c|c|c|c|c|c|c|}
\hline \multirow[b]{2}{*}{ Variables } & \multirow[b]{2}{*}{ Number } & \multicolumn{4}{|l|}{ p53 } & \multicolumn{4}{|l|}{ p21 } & \multicolumn{4}{|l|}{$\mathrm{nm} 23$} & \multicolumn{4}{|l|}{ VEGF } \\
\hline & & Negative & Positive & $\chi^{2}$ & $p$ & Negative & Positive & $\chi^{2}$ & $p$ & Negative & Positive & $\chi^{2}$ & $p$ & Negative & Positive & $\chi^{2}$ & $p$ \\
\hline \multicolumn{18}{|l|}{ Gender } \\
\hline Male & 377 & 149 & 228 & 0.230 & 0.631 & 274 & 103 & 0.670 & 0.413 & 40 & 337 & 0.493 & 0.482 & 85 & 292 & 2.437 & 0.119 \\
\hline Female & 42 & 15 & 27 & & & 33 & 9 & & & 3 & 39 & & & 14 & 28 & & \\
\hline \multicolumn{18}{|l|}{ Age } \\
\hline$\leq 46$ & 229 & 83 & 146 & 1.778 & 0.182 & 157 & 72 & 5.722 & 0.017 & 20 & 209 & 1.282 & 0.258 & 57 & 172 & 0.447 & 0.504 \\
\hline$>46$ & 190 & 81 & 109 & & & 150 & 40 & & & 23 & 167 & & & 42 & 148 & & \\
\hline \multicolumn{18}{|l|}{ Race } \\
\hline Han & 261 & 113 & 148 & 5.014 & 0.025 & 189 & 72 & 0.259 & 0.611 & 32 & 229 & 3.000 & 0.083 & 62 & 199 & 0.006 & 0.937 \\
\hline Minority & 158 & 51 & 107 & & & 118 & 40 & & & 11 & 147 & & & 37 & 121 & & \\
\hline \multicolumn{18}{|c|}{ Smoking status } \\
\hline No & 270 & 109 & 161 & 0.482 & 0.488 & 195 & 75 & 0.425 & 0.514 & 29 & 241 & 0.189 & 0.664 & 72 & 198 & 3.886 & 0.049 \\
\hline Yes & 149 & 55 & 94 & & & 112 & 37 & & & 14 & 135 & & & 27 & 122 & & \\
\hline \multicolumn{18}{|c|}{ Drinking status } \\
\hline No & 250 & 101 & 149 & 0.413 & 0.521 & 180 & 70 & 0.510 & 0.475 & 27 & 223 & 0.194 & 0.659 & 60 & 190 & 0.048 & 0.827 \\
\hline Yes & 169 & 63 & 106 & & & 127 & 42 & & & 16 & 153 & & & 39 & 130 & & \\
\hline \multicolumn{18}{|l|}{ BMI } \\
\hline$\leq 25$ & 348 & 135 & 213 & 0.104 & 0.747 & 249 & 99 & 3.095 & 0.079 & 38 & 310 & 0.963 & 0.327 & 78 & 270 & 1.677 & 0.195 \\
\hline$>25$ & 71 & 29 & 42 & & & 58 & 13 & & & 5 & 66 & & & 21 & 50 & & \\
\hline \multicolumn{18}{|l|}{$\operatorname{AFP}(\mathbf{n g} / \mathrm{ml})^{\mathrm{a}}$} \\
\hline$\leq 400$ & 214 & 92 & 122 & 2.302 & 0.129 & 164 & 50 & 2.007 & 0.157 & 23 & 191 & 0.275 & 0.600 & 55 & 159 & 0.841 & 0.359 \\
\hline$>400$ & 175 & 62 & 113 & & & 123 & 52 & & & 16 & 159 & & & 38 & 137 & & \\
\hline \multicolumn{18}{|c|}{ Child pugh ${ }^{b}$} \\
\hline A & 337 & 127 & 209 & 1.503 & 0.220 & 248 & 88 & 0.425 & 0.514 & 31 & 305 & 1.371 & 0.242 & 77 & 259 & 0.848 & 0.357 \\
\hline B & 56 & 26 & 30 & & & 39 & 17 & & & 8 & 48 & & & 16 & 40 & & \\
\hline \multicolumn{18}{|l|}{ BCLC stage } \\
\hline A & 239 & 93 & 145 & 0.136 & 0.934 & 174 & 64 & 0.693 & 0.707 & 30 & 208 & 3.464 & 0.177 & 55 & 183 & 1.170 & 0.557 \\
\hline B & 69 & 28 & 41 & & & 53 & 16 & & & 4 & 65 & & & 14 & 55 & & \\
\hline C & 111 & 42 & 69 & & & 79 & 32 & & & 9 & 102 & & & 30 & 81 & & \\
\hline No. of tumor & & & & & & & & & & & & & & & & & \\
\hline Single $(n=1)$ & 308 & 119 & 189 & 0.124 & 0.725 & 227 & 81 & 0.111 & 0.739 & 36 & 272 & 2.566 & 0.109 & 76 & 232 & 0.707 & 0.400 \\
\hline $\begin{array}{l}\text { Multiple } \\
(n>1)\end{array}$ & 111 & 45 & 66 & & & 80 & 31 & & & 7 & 104 & & & 23 & 88 & & \\
\hline Tumor size & & & & & & & & & & & & & & & & & \\
\hline$\leq 5 \mathrm{~cm}$ & 138 & 58 & 80 & 0.721 & 0.396 & 110 & 28 & 4.358 & 0.037 & 17 & 121 & 0.945 & 0.331 & 31 & 107 & 0.154 & 0.694 \\
\hline$>5 \mathrm{~cm}$ & 281 & 106 & 175 & & & 197 & 84 & & & 26 & 255 & & & 68 & 213 & & \\
\hline Cirrhosis & & & & & & & & & & & & & & & & & \\
\hline No & 50 & 15 & 34 & 1.640 & 0.200 & 27 & 22 & 9.576 & 0.002 & 7 & 42 & 0.962 & 0.327 & 9 & 40 & 0.868 & 0.351 \\
\hline Yes & 369 & 148 & 221 & & & 280 & 89 & & & 36 & 333 & & & 90 & 279 & & \\
\hline Pathological & $\operatorname{rade}^{c}$ & & & & & & & & & & & & & & & & \\
\hline Well & 24 & 14 & 10 & 3.787 & 0.052 & 22 & 2 & 4.306 & 0.038 & 3 & 21 & 0.196 & 0.658 & 7 & 17 & 0.437 & 0.509 \\
\hline $\begin{array}{l}\text { Moderately/ } \\
\text { Poorly }\end{array}$ & 340 & 130 & 210 & & & 246 & 94 & & & 33 & 307 & & & 79 & 261 & & \\
\hline PVTT & & & & & & & & & & & & & & & & & \\
\hline No & 352 & 141 & 211 & 1.500 & 0.827 & 258 & 94 & 0.070 & 0.999 & 37 & 315 & 2.087 & 0.720 & 79 & 273 & 5.325 & 0.256 \\
\hline Yes & 67 & 3 & 8 & & & 8 & 3 & & & 1 & 10 & & & 1 & 10 & & \\
\hline Radical resec & iond & & & & & & & & & & & & & & & & \\
\hline No & 169 & 89 & 149 & 0.491 & 0.484 & 172 & 66 & 0.420 & 0.517 & 24 & 214 & 0.034 & 0.853 & 63 & 175 & 2.644 & 0.104 \\
\hline Yes & 238 & 69 & 100 & & & 127 & 42 & & & 18 & 151 & & & 33 & 136 & & \\
\hline Antiviral the & apy & & & & & & & & & & & & & & & & \\
\hline No & 276 & 103 & 173 & 1.127 & 0.288 & 187 & 89 & 12.564 & $<0.001$ & 36 & 240 & 6.791 & 0.009 & 65 & 211 & 0.003 & 0.959 \\
\hline Yes & 143 & 61 & 82 & & & 120 & 23 & & & 7 & 136 & & & 34 & 109 & & \\
\hline
\end{tabular}

\section{Association between immunohistochemical markers and OS}

We analyzed the relationship between p53, p21, nm23 and VEGF expression and 2-year, 5-year and overall survival analysis. We found a significant difference in the 2-year survival time of patients with positive and negative VEGF $(P=0.040)$, VEGF-positive patients Death risk was higher than negative (HR= 1.652, 95\% CI=1.024- 2.665) (Table 3). In current study we did not discover these four indicators significant related to the long-term OS of HBV-related HCC (Figure 2 A-D).

\section{Joint effects of immunohistochemical markers and AFP with OS}

The combination of expression of p53, p21, nm23, and VEGF were divided into the relevant groups (Table S1) for assessing the prognostic value 
in HCC according to the associations between the immunohistochemical indicators and OS. As shown in Table 4, the 2-year survival analysis of joint effects were statistically different between group of score $=2$ (p53/VEGF) and group of score $=0$ (p53/VEGF) expression in 419 case tissues $(P=0.047)$, p53/VEGF relative to $\mathrm{p} 53 / \mathrm{VEGF}(+/+)$ was a protective factor for the prognosis of liver cancer $(\mathrm{HR}=0.450,95 \%$ $\mathrm{CI}=0.205-0.988$ ). The 2-year survival analysis of joint effects were statistically different between group of score $=1(\mathrm{P} 21 / \mathrm{NM} 23 / \mathrm{VEGF})$ and group of score $=0$ in 419 case tissues $(P=0.043)$, group of score $=1$ relative to group of score $=0$ was a protective factor for the prognosis of liver cancer (HR=0.477, 95\% $\mathrm{CI}=0.233-0.979)$. The 5-year survival analysis of joint effects were statistically different between group of score $=1(\mathrm{p} 53 / \mathrm{p} 21)$ and group of score $=0$ in 419 case tissues $(P=0.027)$, group of score $=1$ relative to group of score $=0$ is a protective factor for the prognosis of liver cancer $(\mathrm{HR}=0.697,95 \% \mathrm{CI}=0.506-0.960)$. Among the 419 patients, group of the score $=1$ (P21/NM23/VEGF) and group of the score=3 was considerably different from the score $=0$ group ( $P=0.024, P=0.008$, respectively), group of the score $=1$ and group of score $=3$ relative to group of score $=0$ were the protective factor for $\mathrm{HCC}(\mathrm{HR}=0.503$, $95 \% \mathrm{CI}=0.0 .277-0.913 ; \quad \mathrm{HR}=0.179,95 \% \mathrm{CI}=0.050-0.638)$. Overall survival analysis showed that groups with a score of $=3$ (P21NM23VEGF) had lower risk of death than those with a score of $=0(P=0.016, H R=0.311$, $95 \%, \mathrm{CI}=0.120-0.804)$.

Joint effects survival analysis indicated that the 2-year survival time of group of p53/AFP (+/high) were statistically different with group of p53/AFP $(+$ low) $(P=0.038)$ in Table 5 . The risk of death in group p53-VEGF $(+/$ low $)$ group was considerably lower than that in group p53/AFP $(+/$ higher $)(\mathrm{HR}=0.584$, $95 \% \mathrm{CI}=0.352-0.969)$. The 2-year survival time of group of VEGF/AFP (-/low) were statistically different with group of VEGF/AFP (+/high) (P $=0.027)$. The risk of death in group VEGF/AFP (-/low) group was notably lower than that in group VEGF/AFP (+/higher $) \quad(\mathrm{HR}=0.444, \quad 95 \%$ $\mathrm{CI}=0.217-0.910)$. The 5-year survival time of group of VEGF/AFP (+/low) were statistically different with group of VEGF/AFP $(+/$ high $)(P=0.026)$. The risk of death in group VEGF/AFP (+/low) group was remarkably lower than that in group VEGF/AFP $(+/$ higher $)(\mathrm{HR}=0.518,95 \% \mathrm{CI}=0.291-0.923)$.

\section{Stratification analysis}

We further studied VEGF expression with clinical features after 2 years of postoperative stratification analysis after adjusting for drinking status, BCLC stages, PVTT, radical hepatic resection and antiviral treatment (excluding the stratified factor in each stratum) (Figure 3). High VEGF expression could increase the risk of death in non-drinkers, BCLC stage $\mathrm{A}$ and $\mathrm{B}$, non-Antiviral therapy and liver function Child B grade HCC patients $(P=0.040$, $\mathrm{HR}=2.068 ; P=0.041$, HR=2.167; $P=0.034, \mathrm{HR}=1.878$; $P=0.033$, HR $=4$.934; respectively).

Table 2. Clinical features of the patients with HBV-related HCC.

\begin{tabular}{|c|c|c|c|c|}
\hline Variable & $\begin{array}{l}\text { Patients } \\
(n=419)(\%)\end{array}$ & $\begin{array}{l}\text { MST } \\
\text { (months) }\end{array}$ & Log-rank $P$ & HR(95\% CI) \\
\hline \multicolumn{5}{|l|}{ Gender } \\
\hline Male & 377 & 51 & 0.277 & 1 \\
\hline Female & 42 & 80 & & $0.749(0.442-1.268)$ \\
\hline \multicolumn{5}{|l|}{ Age(year) } \\
\hline$\leq 46$ & 229 & 61 & 0.974 & 1 \\
\hline$>46$ & 190 & 51 & & $1.005(0.758-1.331)$ \\
\hline \multicolumn{5}{|l|}{ Race } \\
\hline Han & 261 & 51 & 0.875 & 1 \\
\hline Minority & 158 & 52 & & $1.023(0.764-1.371)$ \\
\hline \multicolumn{5}{|l|}{ Smoking status } \\
\hline No & 270 & 71 & 0.106 & 1 \\
\hline Yes & 149 & 41 & & $1.267(0.949-1.692)$ \\
\hline \multicolumn{5}{|l|}{ Drinking status } \\
\hline No & 250 & 76 & 0.043 & 1 \\
\hline Yes & 169 & 41 & & $1.335(1.006-1.770)$ \\
\hline \multicolumn{5}{|l|}{ BMI } \\
\hline$\leq 25$ & 348 & 52 & 0.918 & 1 \\
\hline$>25$ & 71 & 51 & & $0.981(0.683-1.410)$ \\
\hline \multicolumn{5}{|l|}{$\operatorname{AFP}(\mathrm{ng} / \mathrm{ml})^{\mathrm{a}}$} \\
\hline$\leq 400$ & 214 & 63 & 0.114 & 1 \\
\hline$>400$ & 175 & 42 & & $1.265(0.943-1.697)$ \\
\hline \multicolumn{5}{|l|}{ Child-pugh ${ }^{b}$} \\
\hline A & 337 & 58 & 0.181 & 1 \\
\hline B & 56 & 34 & & $1.291(0.877-1.900)$ \\
\hline \multicolumn{5}{|l|}{ BCLC stage } \\
\hline A & 239 & 123 & $<0.001$ & 1 \\
\hline B & 69 & 95 & & $1.880(1.281-2.758)$ \\
\hline $\mathrm{C}$ & 111 & 29 & & $2.766(2.021-3.786)$ \\
\hline \multicolumn{5}{|l|}{ No. of tumors } \\
\hline Single(n=1) & 308 & 63 & 0.001 & 1 \\
\hline Multiple(n>1) & 111 & 34 & & $1.642(1.219-2.212)$ \\
\hline \multicolumn{5}{|l|}{ Tumor size } \\
\hline$\leq 5 \mathrm{~cm}$ & 138 & 123 & $<0.001$ & 1 \\
\hline$>5 \mathrm{~cm}$ & 281 & 40 & & $1.981(1.416-2.770)$ \\
\hline \multicolumn{5}{|l|}{ Cirrhosis } \\
\hline No & 50 & 88 & 0.191 & 1 \\
\hline Yes & 369 & 51 & & $1.358(0.854-2.158)$ \\
\hline \multicolumn{5}{|l|}{ Pathological grade ${ }^{c}$} \\
\hline Well & 24 & 79 & 0.473 & 1 \\
\hline Moderately/Poorly & 340 & 51 & & $1.276(0.651-2.499)$ \\
\hline \multicolumn{5}{|l|}{ PVTT } \\
\hline No & 352 & 47 & $<0.001$ & 1 \\
\hline Yes & 67 & 40 & & $2.801(2.025-3.875)$ \\
\hline \multicolumn{5}{|l|}{ Radical resection ${ }^{\mathrm{d}}$} \\
\hline No & 169 & 41 & 0.115 & 1 \\
\hline Yes & 238 & 73 & & $1.254(0.944-1.667)$ \\
\hline \multicolumn{5}{|l|}{ Antiviral therapy } \\
\hline No & 276 & 42 & 0.020 & 1 \\
\hline Yes & 143 & NA & & $0.675(0.483-0.945)$ \\
\hline $\begin{array}{l}\text { Notes: a information } \\
\text { information regardin } \\
\text { regarding pathologic } \\
\text { regarding radical res } \\
\text { HCC, hepatocellular } \\
\text { Barcelona Clinic Live } \\
\text { survival time; HR, ha }\end{array}$ & $\begin{array}{l}\text { garding AF } \\
\text { child-pugh } \\
\text { grade was } \\
\text { tion was un } \\
\text { arcinoma; } \\
\text { Cancer; PV } \\
\text { ard ratio; C }\end{array}$ & $\begin{array}{l}\text { level was u } \\
\text { vas unavaila } \\
\text { navailable } \mathrm{f} \\
\text { Ivailable for } \\
\text { II, body mas } \\
\text { T, portal vei } \\
\text { confidence }\end{array}$ & $\begin{array}{l}\text { available for } \\
\text { ble for } 26 \text { pati } \\
\text { r } 55 \text { patients; } \\
12 \text { patients. Hl } \\
\text { s index; AFP, } \\
\text { tumor throm } \\
\text { nterval; NA, n }\end{array}$ & $\begin{array}{l}0 \text { patients; }{ }^{\mathrm{b}} \\
\text { nts; c information } \\
\text { information } \\
\text { V, hepatitis B virus; } \\
\text { I-fetoprotein; BCLC, } \\
\text { bus; MST, median } \\
\text { t available. }\end{array}$ \\
\hline
\end{tabular}


Table 3. Survival analysis between immunohistochemical expression of p53, p21, nm23 and VEGF with 2-year, 5-year and overall survival.

\begin{tabular}{|c|c|c|c|c|c|c|c|}
\hline Variable & $\begin{array}{l}\text { Number } \\
(n=419)\end{array}$ & $\begin{array}{l}2 \text {-year OS } \\
p \text {-value }{ }^{\mathrm{a}}\end{array}$ & $\begin{array}{l}\text { 2-yearOS } \\
\text { HR(95\% CI })^{\text {a }}\end{array}$ & $\begin{array}{l}5 \text {-year } \\
p \text {-value }{ }^{\text {a }}\end{array}$ & $\begin{array}{l}5 \text {-yearOS } \\
\text { HR( }(95 \% \mathrm{CI})^{\text {a }}\end{array}$ & $\begin{array}{l}\text { Overall survival } \\
p \text {-value }{ }^{\mathrm{a}}\end{array}$ & $\begin{array}{l}\text { Overall survival } \\
\text { HR( }(95 \% \text { CI })^{a}\end{array}$ \\
\hline \multicolumn{8}{|l|}{$P 53$} \\
\hline- & 164 & & 1.000 & & & & 1.000 \\
\hline+ & 255 & 0.731 & $1.070(0.729-1.569)$ & 0.410 & $1.142(0.832-1.568)$ & 0.284 & $1.178(0.873-1.591)$ \\
\hline \multicolumn{8}{|l|}{ p21 } \\
\hline- & 307 & & 1.000 & & & & 1.000 \\
\hline+ & 112 & 0.722 & $1.077(0.715-1.623)$ & 0.803 & $0.958(0.686-1.340)$ & 0.997 & $0.999(0.732-1.365)$ \\
\hline \multicolumn{8}{|l|}{$\mathrm{nm} 23$} \\
\hline- & 43 & & 1.000 & & & & 1.000 \\
\hline+ & 376 & 0.779 & $0.913(0.484-1.722)$ & 0.650 & $0.895(0.554-1.445)$ & 0.971 & $0.992(0.631-1.558)$ \\
\hline \multicolumn{8}{|l|}{ VEGF } \\
\hline- & 99 & & 1.000 & & & & 1.000 \\
\hline+ & 320 & 0.040 & $1.652(1.024-2.665)$ & 0.152 & $1.310(0.905-1.896)$ & 0.130 & $1.303(0.925-1.834)$ \\
\hline
\end{tabular}

Notes: aAdjusted for drinking status, BCLC stages, PVTT, radical hepatic resection and adjuvant antiviral treatment. OS, overall survival; HR, hazard ratio; CI, confidence interval.
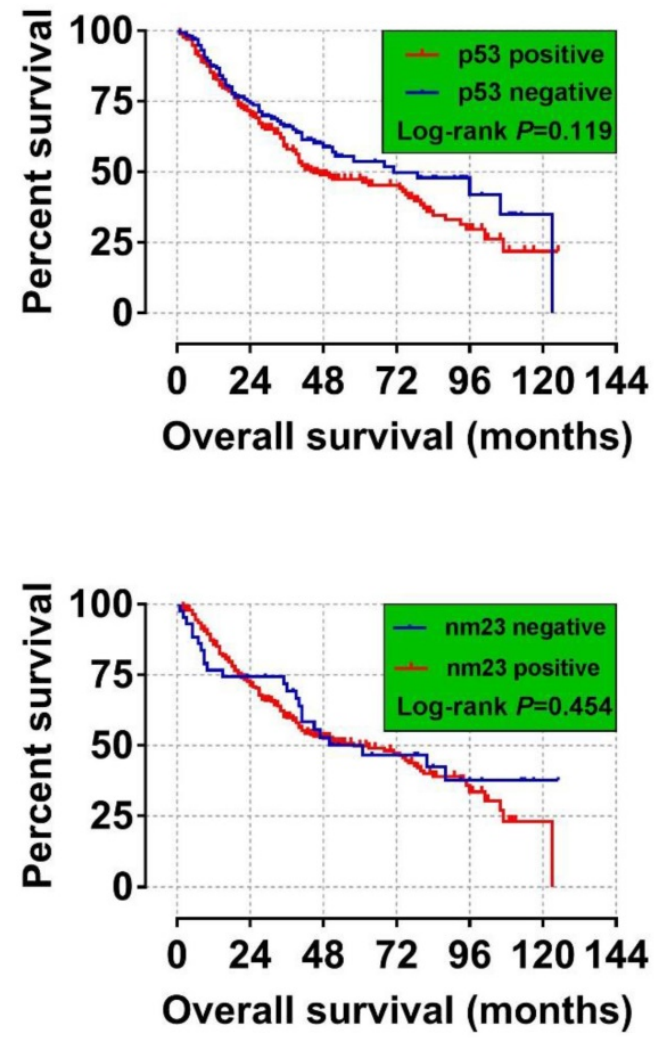
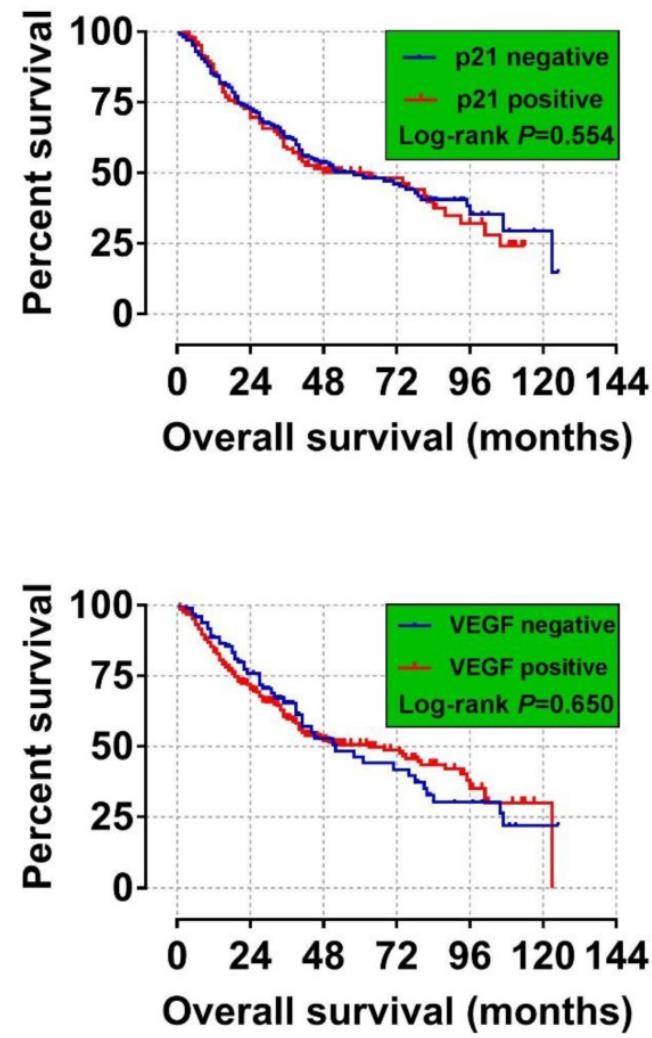

Figure 2. Overall Survival for HCC Patients with Different p53, p21, nm23, and VEGF Expression Statuses. (A) p53; (B) p21;( C) nm23; and (D) VEGF. It was no significant correlation between the four tumor markers and the long-term OS of HBV-associated hepatocellular carcinoma.
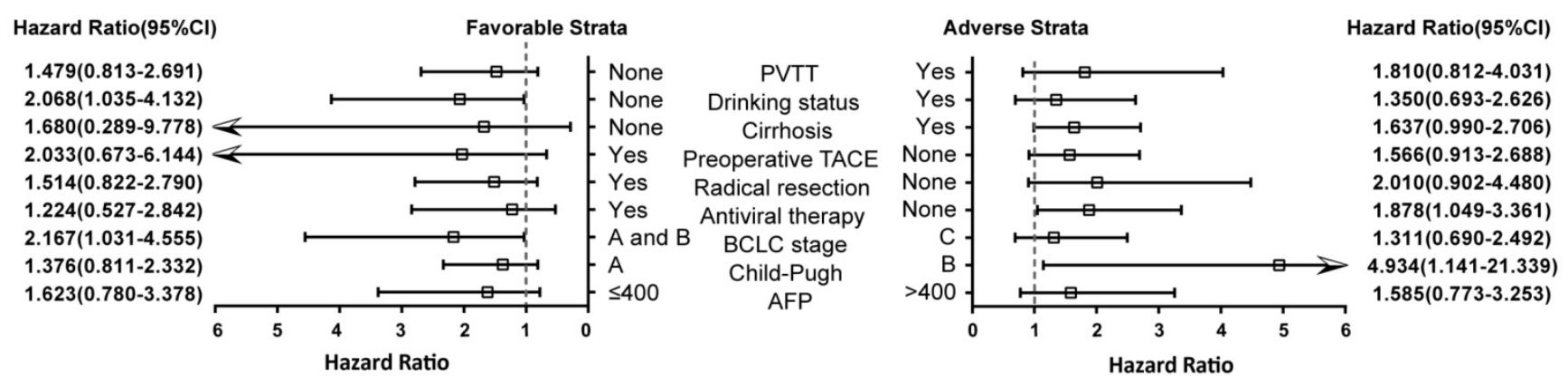

Figure 3. Stratification analysis of the association of VEGF with2-year OS of HBV-related HCC patients. Stratified by favorable and adverse strata. 
Table 4. Joint effects analysis of expression of p53, p21, nm23 and VEGF with 2-year, 5-year and overall survival.

\begin{tabular}{|c|c|c|c|c|c|c|c|}
\hline Variable & $\begin{array}{l}\text { Number } \\
(\mathrm{n}=419)\end{array}$ & $\begin{array}{l}\text { 2-year OS } \\
\text { P-value }\end{array}$ & $\begin{array}{l}\text { 2-year OS } \\
\text { HR( }(95 \% \text { CI })\end{array}$ & $\begin{array}{l}\text { 5-year OS } \\
\text { P-value }\end{array}$ & $\begin{array}{l}\text { 5-year OS } \\
\text { HR( }(95 \% \text { CI })\end{array}$ & $\begin{array}{l}\text { Overall survival } \\
P \text {-value }\end{array}$ & $\begin{array}{l}\text { Overall survival } \\
\text { HR( } 95 \% \text { CI })\end{array}$ \\
\hline \multicolumn{8}{|l|}{ p53/p21 } \\
\hline 0 & 176 & 0.061 & 1.000 & 0.036 & 1.000 & 0.187 & 1.000 \\
\hline 1 & 210 & 0.138 & $0.743(0.502-1.100)$ & 0.027 & $0.697(0.506-0.960)$ & 0.089 & $0.771(0.571-1.041)$ \\
\hline 2 & 33 & 0.200 & $1.500(0.807-2.788)$ & 0.557 & $1.171(0.692-1.981)$ & 0.898 & $1.034(0.619-1.726)$ \\
\hline \multicolumn{8}{|c|}{ p53/nm23 } \\
\hline 0 & 32 & 0.607 & 1.000 & 0.493 & 1.000 & 0.364 & 1.000 \\
\hline 1 & 234 & 0.529 & $1.289(0.585-2.837)$ & 0.750 & $1.095(0.627-1.910)$ & 0.543 & $1.174(0.700-1.970)$ \\
\hline 2 & 153 & 0.858 & $1.077(0.476-2.438)$ & 0.708 & $0.895(0.500-1.600)$ & 0.819 & $0.939(0.547-1.612)$ \\
\hline \multicolumn{8}{|c|}{ p53/VEGF } \\
\hline 0 & 202 & 0.125 & 1.000 & 0.334 & 1.000 & 0.229 & 1.000 \\
\hline 1 & 171 & 0.994 & $0.998(0.680-1.465)$ & 0.291 & $0.840(0.608-1.161)$ & 0.261 & $0.839(0.618-1.139)$ \\
\hline 2 & 46 & 0.047 & $0.450(0.205-0.988)$ & 0.204 & $0.713(0.423-1.201)$ & 0.122 & $0.675(0.410-1.111)$ \\
\hline \multicolumn{8}{|c|}{$\mathrm{p} 21 / \mathrm{nm} 23$} \\
\hline 0 & 31 & 0.317 & 1.000 & 0.237 & 1.000 & 0.468 & 1.000 \\
\hline 1 & 288 & 0.192 & $0.639(0.326-1.252)$ & 0.094 & $0.641(0.380-1.079)$ & 0.266 & $0.748(0.449-1.247)$ \\
\hline 2 & 100 & 0.536 & $0.798(0.390-1.631)$ & 0.247 & $0.717(0.409-1.259)$ & 0.553 & $0.847(0.490-1.464)$ \\
\hline \multicolumn{8}{|c|}{ p21/VEGF } \\
\hline 0 & 225 & 0.421 & 1.000 & 0.189 & 1.000 & 0.319 & 1.000 \\
\hline 1 & 177 & 0.263 & $0.802(0.545-1.18)$ & 0.818 & $0.964(0.705-1.318)$ & 0.800 & $0.962(0.713-1.297)$ \\
\hline 2 & 17 & 0.385 & $0.635(0.228-1.771)$ & 0.068 & $0.389(0.141-1.073)$ & 0.131 & $0.578(0.284-1.178)$ \\
\hline \multicolumn{8}{|c|}{ nm23/VEGF } \\
\hline 0 & 32 & 0.141 & 1.000 & 0.321 & 1.000 & 0.289 & 1.000 \\
\hline 1 & 299 & 0.701 & $0.872(0.435-1.751)$ & 0.687 & $0.894(0.518-1.542)$ & 0.868 & $1.046(0.617-1.773)$ \\
\hline 2 & 88 & 0.133 & $0.538(0.240-1.208)$ & 0.222 & $0.675(0.359-1.269)$ & 0.411 & $0.778(0.427-1.416)$ \\
\hline \multicolumn{8}{|c|}{ p53/p21/nm23 } \\
\hline 0 & 23 & 0.201 & 1.000 & 0.104 & 1.000 & 0.388 & 1.000 \\
\hline 1 & 170 & 0.690 & $0.849(0.379-1.899)$ & 0.462 & $0.797(0.436-1.458)$ & 0.817 & $0.932(0.516-1.685)$ \\
\hline 2 & 196 & 0.335 & $0.674(0.302-1.504)$ & 0.097 & $0.600(0.329-1.096)$ & 0.329 & $0.747(0.416-1.342)$ \\
\hline 3 & 30 & 0.570 & $1.313(0.513-3.362)$ & 0.957 & $1.021(0.49-2.126)$ & 0.912 & $1.041(0.507-2.138)$ \\
\hline \multicolumn{8}{|c|}{ p53/p21/VEGF } \\
\hline 0 & 135 & 0.543 & 1.000 & 0.303 & 1.000 & 0.302 & 1.000 \\
\hline 1 & 198 & 0.413 & $0.840(0.553-1.276)$ & 0.081 & $0.735(0.519-1.039)$ & 0.122 & $0.770(0.553-1.073)$ \\
\hline 2 & 81 & 0.158 & $0.667(0.381-1.17)$ & 0.218 & $0.763(0.497-1.173)$ & 0.257 & $0.791(0.527-1.187)$ \\
\hline 3 & 5 & 0.933 & $1.064(0.250-4.529)$ & 0.376 & $0.523(0.125-2.195)$ & 0.184 & $0.440(0.131-1.479)$ \\
\hline \multicolumn{8}{|c|}{ p21/nm23/VEGF } \\
\hline 0 & 22 & 0.156 & 1.000 & 0.028 & 1.000 & 0.067 & 1.000 \\
\hline 1 & 222 & 0.043 & $0.477(0.233-0.979)$ & 0.024 & $0.503(0.277-0.913)$ & 0.071 & $0.581(0.322-1.048)$ \\
\hline 2 & 160 & 0.058 & $0.498(0.242-1.024)$ & 0.085 & $0.591(0.325-1.075)$ & 0.223 & $0.691(0.381-1.253)$ \\
\hline 3 & 15 & 0.061 & $0.282(0.075-1.061)$ & 0.008 & $0.179(0.050-0.638)$ & 0.016 & $0.311(0.120-0.804)$ \\
\hline \multicolumn{8}{|c|}{ p53/nm23/VEGF } \\
\hline 0 & 25 & 0.198 & 1.000 & 0.448 & 1.000 & 0.300 & 1.000 \\
\hline 1 & 191 & 0.792 & $1.121(0.479-2.624)$ & 0.872 & $1.052(0.569-1.944)$ & 0.556 & $1.194(0.662-2.153)$ \\
\hline 2 & 161 & 0.846 & $1.089(0.460-2.578)$ & 0.668 & $0.871(0.463-1.638)$ & 0.941 & $0.977(0.535-1.786)$ \\
\hline 3 & 42 & 0.167 & $0.447(0.143-1.400)$ & 0.375 & $0.705(0.325-1.527)$ & 0.459 & $0.757(0.362-1.582)$ \\
\hline \multicolumn{8}{|c|}{ p53/p21/nm23/VEGF } \\
\hline 0 & 17 & 0.734 & 1.000 & 0.283 & 1.000 & 0.390 & 1.000 \\
\hline 1 & 137 & 0.378 & $0.675(0.282-1.618)$ & 0.347 & $0.721(0.364-1.426)$ & 0.593 & $0.831(0.421-1.638)$ \\
\hline 2 & 185 & 0.261 & $0.613(0.261-1.439)$ & 0.092 & $0.562(0.287-1.099)$ & 0.261 & $0.683(0.352-1.328)$ \\
\hline 3 & 76 & 0.205 & $0.549(0.217-1.388)$ & 0.240 & $0.650(0.316-1.335)$ & 0.450 & $0.759(0.372-1.551)$ \\
\hline 4 & 4 & 0.407 & $0.405(0.048-3.428)$ & 0.136 & $0.207(0.026-1.645)$ & 0.092 & $0.262(0.055-1.245)$ \\
\hline
\end{tabular}

Notes: a Adjusted for drinking status, BCLC stages, PVTT, radical hepatic resection and adjuvant antiviral treatment. OS, overall survival; HR, hazard ratio; CI, confidence interval; NA, not available.

Table 5. Joint effects analysis between 4 protein and AFP with 2-year, 5-year and overall survival.

\begin{tabular}{|c|c|c|c|c|c|c|c|}
\hline Variables & $\begin{array}{l}\text { Number } \\
(\mathrm{n}=389)^{\mathrm{a}}\end{array}$ & $\begin{array}{l}2 \text {-year OS } \\
p \text {-value }\end{array}$ & $\begin{array}{l}\text { 2-year OS } \\
\text { HR( }(95 \% \text { CI) }\end{array}$ & $\begin{array}{l}5 \text {-year OS } \\
p \text {-value }\end{array}$ & $\begin{array}{l}\text { 5-year OS } \\
\text { HR( }\left(95 \% \text { CI) }{ }^{\text {b }}\right.\end{array}$ & $\begin{array}{l}\text { Overall survival } \\
p \text {-value }{ }^{\mathrm{b}}\end{array}$ & $\begin{array}{l}\text { Overall survival } \\
\text { HR( }(95 \% \mathrm{CI})^{\mathrm{b}}\end{array}$ \\
\hline \multicolumn{8}{|l|}{ p53/AFP } \\
\hline$+/$ high & 113 & 0.222 & & 0.298 & & 0.343 & \\
\hline$-/$ low & 92 & 0.438 & $0.812(0.480-1.374)$ & 0.203 & $0.750(0.481-1.169)$ & 0.248 & $0.777(0.506-1.192)$ \\
\hline$+/$ low & 122 & 0.038 & $0.584(0.352-0.969)$ & 0.110 & $0.724(0.487-1.075)$ & 0.249 & $0.804(0.554-1.165)$ \\
\hline -/high & 62 & 0.345 & $0.760(0.429-1.345)$ & 0.147 & $0.693(0.423-1.137)$ & 0.097 & $0.668(0.415-1.075)$ \\
\hline \multicolumn{8}{|l|}{ p21/AFP } \\
\hline$+/$ high & 52 & 0.504 & & 0.701 & & 0.859 & \\
\hline$-/$ low & 164 & 0.294 & $0.734(0.411-1.309)$ & 0.524 & $0.857(0.534-1.376)$ & 0.833 & $0.952(0.604-1.501)$ \\
\hline$+/$ low & 50 & 0.530 & $0.790(0.378-1.650)$ & 0.463 & $0.803(0.446-1.444)$ & 0.902 & $1.034(0.603-1.774)$ \\
\hline -/high & 123 & 0.958 & $1.015(0.575-1.794)$ & 0.911 & $1.028(0.638-1.656)$ & 0.635 & $1.118(0.706-1.770)$ \\
\hline
\end{tabular}




\begin{tabular}{|c|c|c|c|c|c|c|c|}
\hline Variables & $\begin{array}{l}\text { Number } \\
(n=389)^{a}\end{array}$ & $\begin{array}{l}\text { 2-year OS } \\
p \text {-value }\end{array}$ & $\begin{array}{l}\text { 2-year OS } \\
\text { HR }(95 \% \text { CI })^{\text {b }}\end{array}$ & $\begin{array}{l}5 \text {-year OS } \\
p \text {-value }\end{array}$ & $\begin{array}{l}\text { 5-year OS } \\
\text { HR( } 95 \% \text { CI })^{\text {b }}\end{array}$ & $\begin{array}{l}\text { Overall survival } \\
p \text {-value }\end{array}$ & $\begin{array}{l}\text { Overall survival } \\
\text { HR( }(95 \% \text { CI })^{\mathrm{b}}\end{array}$ \\
\hline \multicolumn{8}{|l|}{ nm23/AFP } \\
\hline +/high & 159 & 0.424 & & 0.647 & & 0.883 & \\
\hline$-/$ low & 23 & 0.373 & $0.581(0.176-1.917)$ & 0.917 & $0.962(0.466-1.986)$ & 0.908 & $1.040(0.535-2.023)$ \\
\hline$+/$ low & 191 & 0.205 & $0.769(0.513-1.154)$ & 0.271 & $0.827(0.590-1.160)$ & 0.536 & $0.903(0.655-1.246)$ \\
\hline -/high & 16 & 0.600 & $1.258(0.533-2.971)$ & 0.708 & $1.145(0.563-2.330)$ & 0.770 & $1.106(0.563-2.171)$ \\
\hline \multicolumn{8}{|l|}{ VEGF/AFP } \\
\hline +/high & 137 & 0.100 & & 0.197 & & 0.130 & \\
\hline$-/$ low & 55 & 0.027 & $0.444(0.217-0.910)$ & 0.133 & $0.672(0.400-1.129)$ & 0.291 & $0.778(0.489-1.239)$ \\
\hline$+/$ low & 38 & 0.164 & $0.605(0.298-1.228)$ & 0.094 & $0.598(0.328-1.092)$ & 0.026 & $0.518(0.291-0.923)$ \\
\hline -/high & 159 & 0.201 & $0.756(0.493-1.161)$ & 0.155 & $0.772(0.540-1.103)$ & 0.182 & $0.792(0.562-1.115)$ \\
\hline
\end{tabular}

Notes: a information regarding AFP level was unavailable for 30 patients ( $\mathrm{n}=389$ ); HR, hazard ratio; CI, confidence interval. ${ }^{\mathrm{b}}$ Adjusted for drinking status, BCLC stages, PVTT ,radical hepatic resection and adjuvant antiviral treatment. OS, overall survival; HR, hazard ratio; CI, confidence interval; NA, not available.

\section{Validation cohort at mRNA level and} bioinformatics analysis

The validation cohort of mRNA dataset were download from the GSE14520. A total of 212 HBV-related HCC were included into validation cohort, and the clinical parameters are summarized in Table S2. Survival analysis suggest that high VEGFA expression significantly linked to poor OS in patients with HBV-related HCC, whereas the other three genes were not showed the statistical significance (Table 6, Figure 4 A-D). Co-expression analysis of VEGFA in HBV-related HCC tumor tissues suggested that VEGFA and its co-expression genes were significant correlated to regulation of cell proliferation, cell growth and apoptotic process, G1/S transition of mitotic cell cycle, cellular response to hypoxia, protein binding, enzyme binding, protein complex assembly, DNA damage checkpoint, Rap1 signaling pathway, HIF-1 signaling pathway, PPAR signaling pathway, cell cycle, biosynthesis of amino acids, and cellular response to hypoxia (Table S3, Figure S1), which were based on the analysis of Gene ontology (GO) and Kyoto Encyclopedia of Genes and Genomes (KEGG) in DAVID v6.8. Prospective molecular mechanisms revealed that high VEGFA expression might participate in the following biological processes and pathways: regulation of transcription from RNA polymerase II promoter in response to hypoxia, regulation of Hypoxia-inducible Factor (HIF) by Oxygen, HIF and HIF1A pathway, and TP53 regulation pathway (Figure 5A-E).

\section{Discussion}

HCC is a highly malignant tumor with poor prognosis. Although the treatment of HCC has important clinical outcomes over the past few decades, the prognosis of HCC patients is still unsatisfactory and has a higher rate of local recurrence and/or distant metastasis. Unfortunately, the prognostic indicators that can guide the treatment of hepatocellular carcinoma are limited, so the survival rate of patients with malignant tumors after surgical resection requires clinicians to participate in the active treatment of relapse and to study the biological and clinicopathological features that reflect tumor behavior.

As we all known, p53, p21, nm23 and VEGF are important biomarkers for diagnosis and assessment the prognosis of HCC. IHC analysis revealed that p53 gene mutations were correlated with the p53 expression and most of HCC tumor tissue with p53 mutations exhibited positive staining for $\mathrm{p} 53$ protein [27]. HCC patients with p53 mutation and up regulated expression in tumor tissue had a shorter OS than those with wild type p53 and low/undetectable p53 expression [28]. However, Chai $Y$ et al. reported p53 expression was not related to cancer characteristics [9]. Prior studies indicated p21 expression was a predictor for clinical performance of patients with HCC, those who had a high p21expression predicted a better survival [17, 29]. However, the predictor value of p21 in HCC patients was affected by HBV proteins and p53 expression [30-33]. Although p53 expression did not link to OS of HBV-related HCC patients in this study, the patients with positive for $\mathrm{p} 53$ expression had higher HR than those negative for p53 expression. p21 expression was associated with some clinical features but not with prognosis of HCC patients. Combined analysis showed p53 and p21 expression levels were associated with 5-year OS. Recent study demonstrated that HCC patients with high VEGF isoforms expression was associated with shorter RFS and poor prognosis [34]. In this study, no significantly difference were found among p53, p21, nm23, VEGF expression level and clinical outcomes of HBV-related HCC patients. We applied different combined analysis in groups using different combinations and our results suggested combination of p53, p23 and VEGF expression might be a good predictor for latter recurrence of HCC patients after hepatectomy. Although previous studies did not apply the combination of these genes as a method of evaluation, our research provided a good research strategy. Further, we needed to collect multiple centers and a larger number of samples to validate our results. 

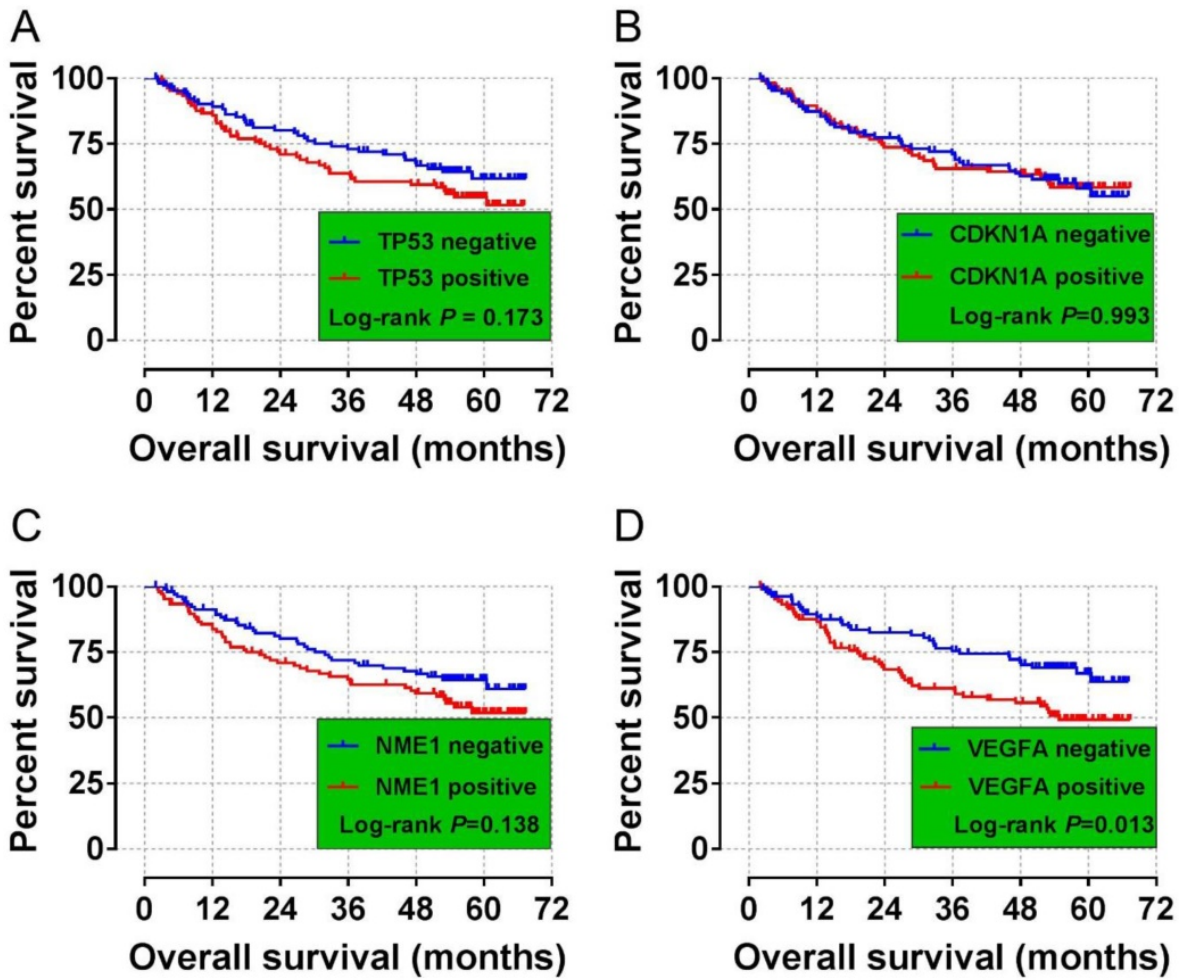

Figure 4. Survival curves for the GSE14520 analyses of HCC patients with different TP53, CDKN1A, NME1, and VEGFA mRNA expression levels. (A) Kaplan-Meier survival curves for OS for different TP53 expression levels. (B) Kaplan-Meier survival curves for the OS analyses of different CDKN1A expression levels. (C) Kaplan-Meier survival curves for the OS analyses of different NMEI expression levels. (D)Kaplan-Meier survival curves for the OS analyses of different VEGFA expression levels.

Table 6. Long-term survival analysis of mRNA expression of TP53, CDKN1A, NME1 and VEGFA in 212 cases of HBV-related HCC in GEO database 14520 data set

\begin{tabular}{|c|c|c|c|c|c|c|}
\hline Gene expression & Patients(n=212) & MST (months) & Crude HR (95\% CI) & Crude P & Adjusted HR (95\% CI) & Adjusted Pa \\
\hline \multicolumn{7}{|l|}{ TP53 } \\
\hline Low & 106 & NA & 1 & & 1 & \\
\hline High & 106 & NA & $1.352(0.874-2.093)$ & 0.175 & $1.081(0.686-1.703)$ & 0.737 \\
\hline \multicolumn{7}{|l|}{ CDKN1A } \\
\hline Low & 106 & NA & 1 & & 1 & \\
\hline High & 106 & NA & $1.002(0.650-1.545)$ & 0.993 & $1.058(0.674-1.662)$ & 0.806 \\
\hline \multicolumn{7}{|l|}{ NME1 } \\
\hline Low & 106 & NA & 1 & & 1 & \\
\hline High & 106 & NA & $1.389(0.898-2.149)$ & 0.140 & $1.316(0.842-2.057)$ & 0.229 \\
\hline \multicolumn{7}{|l|}{ VEGFA } \\
\hline Low & 106 & NA & 1 & & 1 & \\
\hline High & 106 & 54 & $1.735(1.115-2.699)$ & 0.015 & $1.651(1.035-2.634)$ & 0.035 \\
\hline
\end{tabular}

AFP is one of the most commonly used biomarkers in the diagnosis and evaluation of clinical outcomes of HBV related HCC $[5,35]$. However, there is still some controversy over the prognostic value of AFP $[12,14,36,37]$. In this study, we attempted to perform a joint analysis of AFP and immunohistochemical markers to explore whether such conjoint analysis could improve the predictive efficacy of clinical outcomes. In this report, 4 immunohistochemical indicators were combined with AFP level for analysis of clinical outcomes of
HBV-related HCC patients. We found that p53 positive patients with low levels of AFP had better 2 years survival than those who both high levels of AFP and p53 positive. Compared to patients with VEGF positive and high level of AFP, VEGF negative and low level of AFP patients had a good two years of survival, and patients with VEGF positive and low level of AFP had a good five-year survival time. AFP was not a strong prognostic marker despite the fact that serum AFP level above $400 \mathrm{ng} / \mathrm{ml}$ would indicate poor overall survival time after hepatectomy in 
patients with HBV-associated HCC [38]. Some studies reported that combined analysis enhanced diagnosis and prognosis value of AFP in HCC [39, 40]. Our results suggested joint analysis of AFP, p53 and VEGF might be performed for the prediction of the clinical outcomes of HBV-related HCC patients in Guangxi.

As shown in previous studies [4, 7, 41, 42], although four indicators had an effect on the prognosis of patients with hepatocellular carcinoma after surgery, joint analysis was less. This research attempted to study the relationship between the combination of four immunohistochemical indicators and the prognosis. We found that the patients with three proteins combined with P21, NM23, and VEGF, the group of score $=1$ and group of score $=3$ had a longer survival time in 5 years than group of score $=0$.
Any protein that gives a score greater than 0 was a protective factor for the prognosis. However, for 2 years and overall survival, the statistical $P$ values were near 0.05 , and perhaps increasing the sample size might show statistical difference.

Previous study had shown that VEGFA expression could be activated by transcription of various transcription factors, including Sp1, NFkB, AP1 and HIF-1a. HIF-1a could inhibit VEGFA expression, whereas VEGF-mediated upregulation of IL-6 triggers the progression of hemangioma cells [43]. We found through the functional enrichment of VEGFA and its co-expression related genes that VEGFA affects tumors basic cell states by participating in the regulation of cell proliferation, cell cycle, apoptosis.
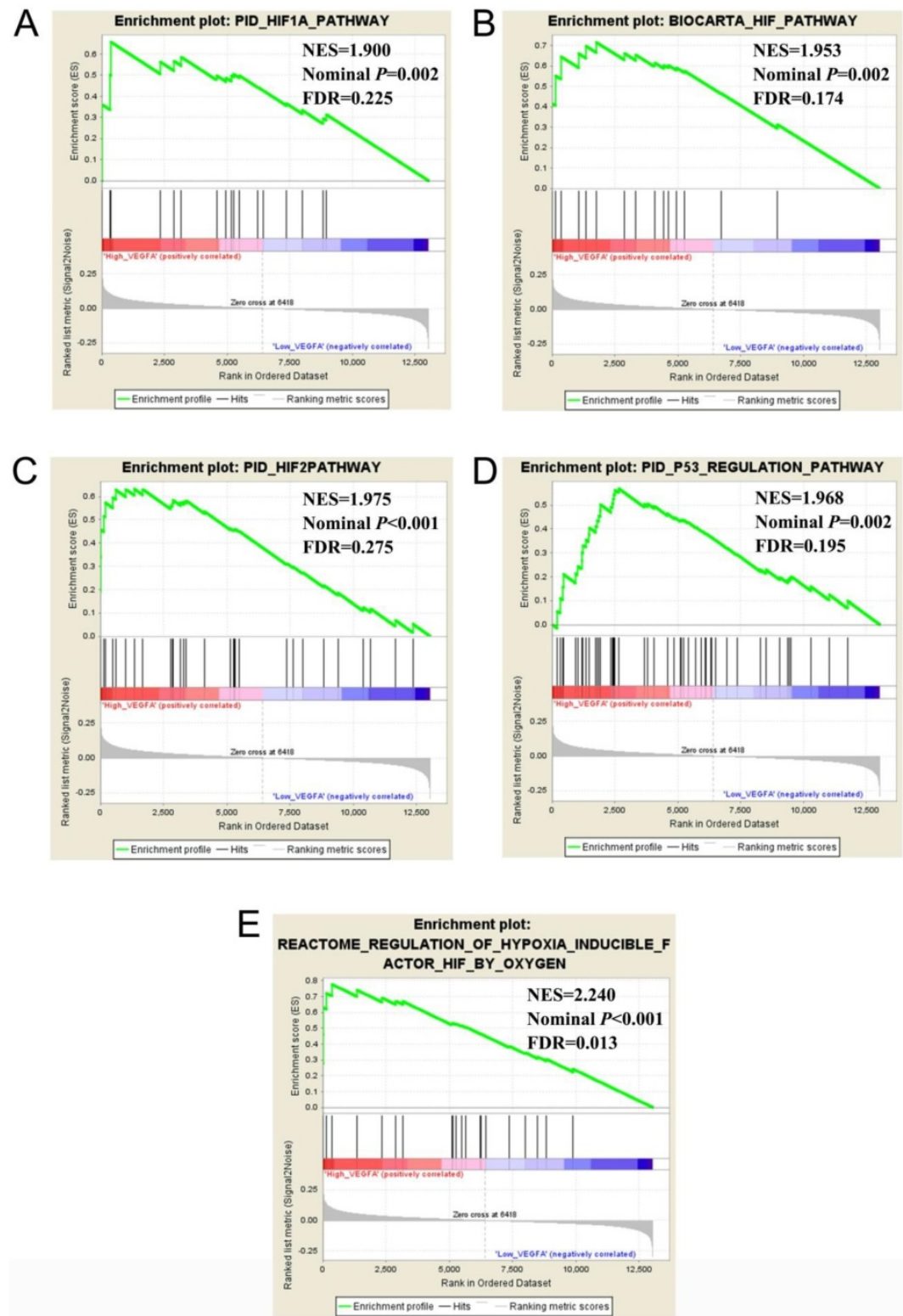

Figure 5. Comparative gene expression studies of HCC tumors and adjacent normal samples in GSE14520 dataset using GSEA. Notes: GSEA results of c2 (A-D) and c5 (E) Abbreviations: NES, Normalized enrichment score; FDR, false discovery rate. 
Our research had certain limitations that needed to be recognized. First, because of the small sample size, the prognosis of many immunohistochemical markers did not reach statistical significance. Second, our sample size was not large enough to verify the impact of rare levels on OS in stratified analysis. Third, our samples came from HBV positive HCC in Guangxi, and our results required larger samples and multicenter validation. Fourth, since the molecular mechanism of VEGFA in this study was explored by GSEA, the validation of in vitro and in vivo experiments was lacking. Therefore, our results still need to be experimentally verified in future study.

Despite these limitations, our study was the first to predict the prognosis of HBV-related HCC using four immunohistochemical indicators and AFP assessment. Our results suggested that the four immunohistochemical indicators had some clinical value in predicting the prognosis of HCC. The prognostic value of the four immunohistochemical indicators and AFP in HBV-related HCC patients could be enhanced using combined and stratified analysis.

\section{Conclusion}

In conclusion, our findings demonstrated that expression of VEGF may play the role as a prognostic indicator for patients with HBV-related HCC. The prospective molecular mechanism of VEGF might involve in the biological processes and pathways of hypoxia, cell cycle, cell apoptosis, cell proliferation and DNA damage checkpoint, which were importance for the base status of normal cells.

\section{Supplementary Material}

Supplementary figures and tables.

http://www.jcancer.org/v11p0906s1.pdf

\section{Acknowledgments}

This work was supported in part by the National Natural Science Foundation of China (No.: 81560535, 81802874, 81072321, 30760243, 30460143 and 30560133), Natural Science Foundation of Guangxi Province of China (Grant No.2017JJB140189y, 2018GX NSFAA050119), 2009 Program for New Century Excellent Talents in University (NCET), Guangxi Natural Sciences Foundation (No.: GuiKeGong 1104003A-7), and Guangxi Health Ministry Medicine Grant (Key-Scientific Research-Grant Z201018). The present study is also partly supported by Scientific Research Fund of the Health and Family Planning Commission of Guangxi Zhuang Autonomous Region (Z2016318, Z2016307), Key laboratory of HighIncidence-Tumor Prevention \& Treatment (Guangxi Medical University), Ministry of Education (GKE20
18-01, GKE2019-11), the Guangxi Key R \& D Program (GKEAB18221019), The Basic Ability Improvement Project for Middle-aged and Young Teachers in Colleges and Universities in Guangxi (2018KY0110), Innovation Project of Guangxi Graduate Education (JGY2018037), and 2018 Innovation Project of Guangxi Graduate Education (YCBZ2018036). As well as, the present study is also partly supported by Guangxi Key Laboratory for the Prevention and Control of Viral Hepatitis (No. GXCDCKL201902) and Research Institute of Innovative Think-tank in Guangxi Medical University (The gene-environment interaction in hepatocarcinogenesis in Guangxi HCCs and its translational applications in the HCC prevention). We would also acknowledge the supported by the Key laboratory of High-Incidence-Tumor Prevention \& Treatment (Guangxi Medical University), Ministry of Education. The authors thank the contributors of GSE14520 (https://www.ncbi.nlm.nih.gov/geo/que ry/acc.cgi?acc $=G S E 14520$ ) for sharing the HBVrelated HCC dataset on open access. The authors thank Prof. Xiao Qin, Xigang Chen, Bin Chen, Zhixiong Su, Ming Su, Zhang Wen, Jingning Lu, Ning Peng, Hai Zhu for providing part of hepatocellular carcinoma samples for this study, who are from the Department of Hepatobiliary Surgery, the First Affiliated Hospital of Guangxi Medical University. Thanks also go to the researcher Jiaquan Li and Ying Gui from Guangxi Medical University for their contribution to specimen management.

\section{Competing Interests}

The authors have declared that no competing interest exists.

\section{References}

1. Torre LA, Bray F, Siegel RL, Ferlay J, Lortet-Tieulent J, Jemal A. Global cancer statistics, 2012. CA: a cancer journal for clinicians. 2015; 65: 87-108.

2. Abdou AG, Abd-Elwahed M, Badr M, Helmy M, Soliman EA, Maher D. The Differential Immunohistochemical Expression of p53, c-Jun, c-Myc, and p21 Between HCV-related Hepatocellular Carcinoma With and Without Cirrhosis. Applied immunohistochemistry \& molecular morphology : AIMM. 2016; 24: $75-87$.

3. An R, Meng J, Shi Q, Dai XX, Chen JH, Lei YJ, et al. Expressions of nucleoside diphosphate kinase (nm23) in tumor tissues are related with metastasis and length of survival of patients with hepatocellular carcinoma. Biomedical and environmental sciences : BES. 2010; 23: 267-72.

4. Attallah AM, El-Far M, Abdelrazek MA, Omran MM, Attallah AA, Elkhouly AA, et al. Combined use of nuclear phosphoprotein c-Myc and cellular phosphoprotein p53 for hepatocellular carcinoma detection in high-risk chronic hepatitis C patients. British journal of biomedical science. 2017; 74: $170-5$

5. Marrero JA, Kulik LM, Sirlin CB, Zhu AX, Finn RS, Abecassis MM, et al. Diagnosis, Staging, and Management of Hepatocellular Carcinoma: 2018 Practice Guidance by the American Association for the Study of Liver Diseases. Hepatology. 2018; 68: 723-50.

6. Buijs $\mathrm{N}$, Oosterink JE, Jessup $\mathrm{M}$, Schierbeek $\mathrm{H}$, Stolz DB, Houdijk AP, et al. A new key player in VEGF-dependent angiogenesis in human hepatocellular carcinoma: dimethylarginine dimethylaminohydrolase 1. Angiogenesis. 2017; 20: 557-65.

7. Campagnolo L, Telesca C, Massimiani M, Stuhlmann H, Angelico M, Lenci I et al. Different expression of VEGF and EGFL7 in human hepatocellular carcinoma. Digestive and liver disease : official journal of the Italian Society of Gastroenterology and the Italian Association for the Study of the Liver. 2016; 48: 76-80. 
8. Cao G, Li X, Qin C, Li J. Prognostic Value of VEGF in Hepatocellular Carcinoma Patients Treated with Sorafenib: A Meta-Analysis. Medical science monitor : international medical journal of experimental and clinical research. 2015; 21: 3144-51.

9. Chai Y, Xiaoyu L, Haiyan W. Correlation between expression levels of PTEN and p53 genes and the clinical features of HBsAg-positive liver cancer. Journal of BUON : official journal of the Balkan Union of Oncology. 2017; 22: 942-6.

10. Du P, Xu B, Zhang D, Shao Y, Zheng X, Li X, et al. Hierarchical investigating the predictive value of $\mathrm{p} 53, \mathrm{COX} 2, \mathrm{EGFR}, \mathrm{nm} 23$ in the post-operative patients with colorectal carcinoma. Oncotarget. 2017; 8: 954-66.

11. Gerdes H. The p53 gene and hepatocellular carcinoma. Gastroenterology. 1991; 101: 1444-5.

12. Giannini EG, Marenco S, Borgonovo G, Savarino V, Farinati F, Del Poggio P, et al. Alpha-fetoprotein has no prognostic role in small hepatocellular carcinoma identified during surveillance in compensated cirrhosis. Hepatology. 2012; 56: 1371-9.

13. Goldenberg D, Eferl R. p21Waf1/Cip1 revisited: oncogenic function in hepatocellular carcinoma. Gut. 2014; 63: 1372-3.

14. Gomez-Rodriguez R, Romero-Gutierrez M, Artaza-Varasa T, Gonzalez-Frutos C, Ciampi-Dopazo JJ, de-la-Cruz-Perez G, et al. The value of the Barcelona Clinic Liver Cancer and alpha-fetoprotein in the prognosis of hepatocellular carcinoma. Revista espanola de enfermedades digestivas : organo oficial de la Sociedad Espanola de Patologia Digestiva. 2012; 104: 298-304.

15. Graur F, Furcea L, Mois E, Biliuta A, Rozs AT, Negrean V, et al. Analysis of p53 Protein Expression in Hepatocellular Carcinoma. Journal of gastrointestinal and liver diseases : JGLD. 2016; 25: 345-9.

16. Kondo K, Chijiiwa K, Kai M, Otani K, Nagaike K, Ohuchida J, et al. Surgical strategy for hepatocellular carcinoma patients with portal vein tumor thrombus based on prognostic factors. Journal of gastrointestinal surgery : official journal of the Society for Surgery of the Alimentary Tract. 2009; 13: 1078-83.

17. Kao JT, Chuah SK, Huang CC, Chen CL, Wang CC, Hung $\mathrm{CH}$, et al. $\mathrm{P} 21 / \mathrm{WAF} 1$ is an independent survival prognostic factor for patients with hepatocellular carcinoma after resection. Liver international : official journal of the International Association for the Study of the Liver. 2007; 27: 772-81.

18. Lee SH, Lee JS, Na GH, You YK, Kim DG. Immunohistochemical markers for hepatocellular carcinoma prognosis after liver resection and liver transplantation. Clinical transplantation. 2017; 31.

19. Li XR, Liu M, Zhang YJ, Wang JD, Zheng YQ, Li J, et al. ER, PgR, HER-2, Ki-67, topoisomerase IIalpha, and $\mathrm{nm} 23-\mathrm{H} 1$ proteins expression as predictors of pathological complete response to neoadjuvant chemotherapy for locally advanced breast cancer. Medical oncology. 2011; 28 Suppl 1: S48-54.

20. Mao CS, Yin H, Ning HB, Peng Z, Li K, Ding GQ. Levels of HBx, VEGF, and CEACAM1 in HBV-related hepatocellular carcinoma and their correlation with cancer prognosis. European review for medical and pharmacological sciences. 2017; 21: 3827-33.

21. Liao X, Liu X, Yang C, Wang X, Yu T, Han C, et al. Distinct Diagnostic and Prognostic Values of Minichromosome Maintenance Gene Expression in Patients with Hepatocellular Carcinoma. Journal of Cancer. 2018; 9: 2357-73.

22. Subramanian A, Tamayo P, Mootha VK, Mukherjee S, Ebert BL, Gillette MA, et al. Gene set enrichment analysis: a knowledge-based approach for interpreting genome-wide expression profiles. Proceedings of the National Academy of Sciences of the United States of America. 2005; 102: 15545-50.

23. Liberzon A, Birger C, Thorvaldsdottir H, Ghandi M, Mesirov JP, Tamayo P. The Molecular Signatures Database (MSigDB) hallmark gene set collection. Cell systems. 2015; 1: 417-25.

24. Huang da W, Sherman BT, Lempicki RA. Bioinformatics enrichment tools: paths toward the comprehensive functional analysis of large gene lists. Nucleic acids research. 2009; 37: 1-13.

25. Huang da W, Sherman BT, Lempicki RA. Systematic and integrative analysis of large gene lists using DAVID bioinformatics resources. Nature protocols. 2009; 4: 44-57.

26. Maere S, Heymans K, Kuiper M. BiNGO: a Cytoscape plugin to assess overrepresentation of gene ontology categories in biological networks. Bioinformatics. 2005; 21: 3448-9.

27. Qi LN, Bai T, Chen ZS, Wu FX, Chen YY, De Xiang B, et al. The p53 mutation spectrum in hepatocellular carcinoma from Guangxi, China : role of chronic hepatitis B virus infection and aflatoxin B1 exposure. Liver international : official journal of the International Association for the Study of the Liver. 2015; 35: 999-1009.

28. Liu J, Ma Q, Zhang M, Wang X, Zhang D, Li W, et al. Alterations of TP53 are associated with a poor outcome for patients with hepatocellular carcinoma: evidence from a systematic review and meta-analysis. European journal of cancer. 2012; 48: 2328-38

29. Zhang MF, Zhang ZY, Fu J, Yang YF, Yun JP. Correlation between expression of p53, p21/WAF1, and MDM2 proteins and their prognostic significance in primary hepatocellular carcinoma. Journal of translational medicine. 2009; 7: 110.

30. Yano M, Ohkoshi S, Aoki YH, Takahashi H, Kurita S, Yamazaki K, et al. Hepatitis $B$ virus $X$ induces cell proliferation in the hepatocarcinogenesis via up-regulation of cytoplasmic p21 expression. Liver international : official journal of the International Association for the Study of the Liver. 2013; 33: 1218-29.
31. Xu J, Liu H, Chen L, Wang S, Zhou L, Yun X, et al. Hepatitis B virus X protein confers resistance of hepatoma cells to anoikis by up-regulating and activating p21-activated kinase 1. Gastroenterology. 2012; 143: 199-212 e4.

32. Qin LF, Ng IO, Fan ST, Ng M. p21/WAF1, p53 and PCNA expression and p53 mutation status in hepatocellular carcinoma. International journal of cancer. 1998; 79: 424-8.

33. Hsu YL, Kuo PL, Chiang LC, Lin CC. Involvement of p53, nuclear factor kappaB and Fas/Fas ligand in induction of apoptosis and cell cycle arrest by saikosaponin d in human hepatoma cell lines. Cancer letters. 2004; 213: 213-21.

34. Zhuang PY, Shen J, Zhu XD, Lu L, Wang L, Tang ZY, et al. Prognostic roles of cross-talk between peritumoral hepatocytes and stromal cells in hepatocellular carcinoma involving peritumoral VEGF-C, VEGFR-1 and VEGFR-3. PloS one. 2013; 8: e64598.

35. Parpart S, Roessler S, Dong F, Rao V, Takai A, Ji J, et al. Modulation of miR-29 expression by alpha-fetoprotein is linked to the hepatocellular carcinoma epigenome. Hepatology. 2014; 60: 872-83.

36. Shim JH, Yoon DL, Han S, Lee YJ, Lee SG, Kim KM, et al. Is serum alpha-fetoprotein useful for predicting recurrence and mortality specific to hepatocellular carcinoma after hepatectomy? A test based on propensity scores and competing risks analysis. Annals of surgical oncology. 2012; 19: 3687-96.

37. Toyoda H, Kumada T, Kaneoka Y, Osaki Y, Kimura T, Arimoto A, et al. Prognostic value of pretreatment levels of tumor markers for hepatocellular carcinoma on survival after curative treatment of patients with HCC. Journal of hepatology. 2008; 49: 223-32.

38. Yang SL, Liu LP, Yang S, Liu L, Ren JW, Fang X, et al. Preoperative serum alpha-fetoprotein and prognosis after hepatectomy for hepatocellular carcinoma. The British journal of surgery. 2016; 103: 716-24.

39. Wang X, Zhang W, Liu Y, Gong W, Sun P, Kong X, et al. Diagnostic value of prothrombin induced by the absence of vitamin $\mathrm{K}$ or antagonist-II (PIVKA-II) for early stage HBV related hepatocellular carcinoma. Infectious agents and cancer. 2017; 12: 47.

40. Kamiyama T, Orimo T, Wakayama K, Shimada S, Nagatsu A, Yokoo H, et al. Survival outcomes of hepatectomy for stage B Hepatocellular carcinoma in the BCLC classification. World journal of surgical oncology. 2017; 15: 156.

41. Liu YB, Gao SL, Chen XP, Peng SY, Fang HQ, Wu YL, et al. Expression and significance of heparanase and $\mathrm{nm} 23-\mathrm{H} 1$ in hepatocellular carcinoma. World journal of gastroenterology. 2005; 11: 1378-81.

42. Zhang Y, Liu Y, Duan J, Yan H, Zhang J, Zhang H, et al. Hippocalcin-like 1 suppresses hepatocellular carcinoma progression by promoting p21(Waf/Cip1) stabilization by activating the ERK1/2-MAPK pathway. Hepatology. 2016; 63: 880-97.

43. Fu X, Zhai S, Yuan J. Interleukin-6 (IL-6) triggers the malignancy of hemangioma cells via activation of HIF-1alpha/VEGFA signals. European journal of pharmacology. 2018; 841: 82-9. 